\title{
Les doctrines antiques des figures : quelques idées reçues
}

About a Few Prejudices Concerning Ancient Theories of Figures

\section{Pierre Chiron}

\section{(2) OpenEdition \\ 1 Journals}

\section{Édition électronique}

URL : http://journals.openedition.org/pratiques/2462

DOI : 10.4000/pratiques.2462

ISSN : 2425-2042

Éditeur

Centre de recherche sur les médiations (CREM)

\section{Référence électronique}

Pierre Chiron, «Les doctrines antiques des figures : quelques idées reçues », Pratiques [En ligne], 165-166 | 2015, mis en ligne le 01 octobre 2015, consulté le 14 novembre 2019. URL : http:// journals.openedition.org/pratiques/2462; DOI : 10.4000/pratiques.2462

Ce document a été généré automatiquement le 14 novembre 2019.

(c) Tous droits réservés 


\title{
Les doctrines antiques des figures : quelques idées reçues
}

\author{
About a Few Prejudices Concerning Ancient Theories of Figures
}

\author{
Pierre Chiron
}

1 Avant de dire quels sont les préjugés à combattre à propos non pas de la théorie ancienne des figures mais des très diverses doctrines élaborées par nos lointains collègues et cela sur une très longue durée, nous voudrions préciser d'abord de quelle manière nous abordons ces questions.

2 1) D'abord en tant que philologue, dont la tâche consiste à élaborer des éditions critiques, à partir de l'étude scientifique des rapports de filiation entre les manuscrits conservés. Le but est archéologique, en quelque sorte : il s'agit de retrouver le texte original ou, plus souvent, de reconstituer le texte le plus proche possible du texte original.

3 En l'occurrence, il s'agit de traités de rhétorique grecs. Or, du point de vue de l'éditeur, le corpus rhétorique - par rapport au théâtre, à la poésie, à l'histoire ou à la philosophie - offre deux caractéristiques assez particulières, l'une plutôt positive, l'autre négative.

4 La rhétorique souffre depuis le Gorgias de Platon d'un préjugé défavorable toujours vivace, et cela tend à expliquer les retards dans l'étude des textes, y compris à l'époque moderne. Pour certains philosophes, aujourd'hui encore, et malgré le fait qu'Aristote lui-même a écrit un traité de rhétorique, cette discipline ne mérite pas plus de considération que la sophistique, d'où ce paradoxe, eu égard à l'importance capitale de la parole publique dans les sociétés anciennes, qui veut que le corpus rhétorique dont nous avons hérité est un champ de recherche exploré depuis assez peu de temps.

On connait les origines formalistes de cette redécouverte, puis le rôle joué par J. Austin au milieu des années 1950, avec la pragmatique qui, par bien des côtés, retrouve la perspective rhétorique ancienne. Une autre étape de ce processus est incarnée par C. Perelman à la fin des années 1950, Perelman qui a rendu son ampleur à l'« empire rhétorique ». On se souvient de l'intérêt plus ponctuel que lui ont porté des 
sémiologues comme R. Barthes dans la décennie suivante, puis les tenants de la linguistique structurale ou des herméneutes, tel P. Ricœur dans ses recherches sur la métaphore.

6 Cet élan de curiosité n'a pas suffi à provoquer le nécessaire travail de mise à jour des connaissances historicophilologiques. La Société internationale d'histoire de la rhétorique n'a commencé ses activités qu'à la fin des années 1970, en 1977 exactement, et l'exhumation des traités dans la collection Budé n'a vraiment commencé qu'avec les années 1980 et elle est encore en cours actuellement. Pendant très longtemps, les références des spécialistes des théories du langage ont été soit aux classiques français, soit au corpus latin sur lequel il repose en partie, pour l'essentiel Cicéron et Quintilien, soit - pour le grec - à des recueils allemands de qualité très inégale datant du milieu du $\mathrm{XIX}^{\mathrm{e}}$ ou du début du XXe , sans traduction ni commentaire français.

7 À toute chose malheur est bon: cette exhumation tardive se fait dans des conditions scientifiques meilleures et les éditions récentes présentent la plupart du temps une fiabilité excellente. De plus, cette exhumation coïncide, ce qui est très positif, avec un courant très puissant de l'historiographie, qui est la contestation de l'idée ancienne de décadence de l'Empire romain, notamment par l'historien anglo-saxon P. Brown (son ouvrage The Making of Late Antiquity date de $1976^{1}$ ). Les textes redécouverts, qui sont souvent des textes de l'époque impériale, bénéficient donc d'une double résurrection, rhétorique et historique.

8 Le corollaire d'un tel retard est qu'il reste un gros travail à effectuer pour diffuser les résultats de cette recherche récente. Et si nous avons laissé entendre dans le titre cidessus qu'il y a un certain nombre de malentendus concernant la rhétorique ancienne, ce n'est un reproche pour personne, cela tient au fait que la connaissance des textes n'a progressé que récemment. On peut dire par exemple que la documentation dont disposait un R. Barthes, ou même un historien de l'éducation antique comme $\mathrm{H}$. Marrou, est désormais obsolète. C'est aussi la raison pour laquelle les recueils, lexiques et autres dictionnaires des figures que l'on publie à jet continu ont une partie historique généralement très faible et dépendent in fine des recueils français des XVIII ${ }^{\mathrm{e}}$ ou XIX ${ }^{e}$ siècles français. Au risque de tomber dans la caricature, on peut citer l'exemple de la fiche "figure de style » de l'encyclopédie en ligne Wikipedia. On y lit ceci : "Les figures de style constituent un vaste ensemble complexe de procédés variés et à l'étude délicate. Les spécialistes ont identifié, depuis l'Antiquité gréco-romaine (avec Cicéron, Quintilien) des centaines de figures de style et leur ont attribué des noms savants, puis ont tenté de les classer (Fontanier, Dumarsais) $»^{2}$. On constate que la reconnaissance de la complexité du dossier va de pair avec une grande ignorance des sources et notamment, la totale occultation de la tradition grecque.

9 Cette tradition originelle, la plus intéressante à nos yeux sur le plan théorique avant les développements plus récents soutenus par la science linguistique, est donc largement méconnue. Et il ne s'agit pas de quelques textes d'importance mineure. Pour la technique rhétorique, qui englobe un substantiel matériau relatif aux figures, ce ne sont pas moins de 20 volumes Budé, sur un total prévisible de 25 , qui ont été publiés récemment et dont la lecture est encore trop peu répandue chez les historiens des théories du langage.

10 Mais le défaut du corpus rhétorique, philologiquement parlant, est que, en raison de son caractère technique, sa transmission a été opérée par des utilisateurs, des savants ou des professeurs : la datation, l'authentification des textes est rendue difficile par ce 
qu'on appelle pudiquement " tradition fluide ». Il existe un grand nombre de textes que le copiste a amalgamés, ou corrigés, ou complétés, pour les adapter à ses cours ou à son usage personnel. L'un des meilleurs traités des figures de l'époque impériale, le traité d'Alexandros (Spengel, $1856: 7-40$ ) - qui par parenthèse n'a pas encore été traduit en français -, a fait l'objet d'une réécriture dans laquelle les exemples païens ont été remplacés par des illustrations tirées d'un père de l'Église, Grégoire de Nazianze. Cette réécriture a elle-même été abrégée, ce qui fait qu'on dispose non seulement d'une version païenne et d'une version chrétienne, mais d'une version longue et d'une version courte de l'Alexandros chrétien. Autre exemple: le traité Des Figures d'un auteur du nom de Zonaios (Spengel, 1856 : 161-170) n'est peut-être en réalité qu'un faux forgé au Xvie siècle par l'un des copistes au service du cardinal de Lorraine à la cour de Fontainebleau, Constantin Paléocappa (Conley, 2004). Autre exemple : nous avons eu l'occasion de travailler sur un traité $\mathrm{Du}$ Style (Démétrios, 1993) qui est daté par certains du III e siècle avant J.-C. et par d'autres (par exemple N. Marini, voir Démétrios, 2007) du second siècle après. Un grand nombre de textes sont donc difficiles à attribuer et à dater et ils sont même souvent constitués de parties hétérogènes d'auteurs et d'époques différentes, réunies, ou recomposées pour les besoins d'autodidactes ou de professeurs.

11 En somme, et c'est par là que nous voulions commencer, le dossier des théories anciennes des figures, comme en général le dossier de la rhétorique ancienne, couvre un domaine à la fois récemment redécouvert, et donc encore largement ignoré, et extrêmement difficile à instruire, à cause des modalités de sa transmission.

2) En dehors de la connaissance des textes, qui se justifie d'elle-même, quelle attitude avoir face à ces théories récemment exhumées? Il importe là aussi de définir clairement les options que l'on prend. Les sciences de l'Antiquité, au xxe siècle, ont été l'un des principaux domaines d'application de l'anthropologie structurale. L'un des efforts permanents de chercheurs comme J.-P. Vernant a été de défaire la connivence subjective avec l'Antiquité grecque, de ridiculiser l'admiration béate pour le prétendu miracle grec, afin d'atteindre de bonnes conditions d'objectivité, et de mettre toutes les sciences humaines au service de la connaissance complète d'un moment de l'histoire occidentale, certes très riche, mais qui ne doit pas être érigé en modèle indépassable.

13 En clair, les figures dont nous héritons de l'Antiquité n'ont pas à être sacralisées, ni discréditées, d'ailleurs, à priori, et dans le même temps leur description, leur théorisation doit être resituée dans le contexte qui leur a donné naissance, historicisées en quelque sorte, en donnant à ce terme toute l'ampleur que lui donnent les anthropologues, c'est-à-dire en tâchant de relier entre eux tous les aspects de la vie humaine, aspects politiques, culturels, juridiques, etc. Nous insisterons beaucoup par exemple, dans la suite de cet exposé, sur les liens entre figures et philosophie, entre figures et éducation.

14 En somme, nous pensons qu'une histoire sérieuse des figures doit comporter tous les éléments de contexte qui permettent de comprendre les raisons de leur thématisation. Ce n'est pas tout à fait la contextualisation à laquelle réfère le titre du présent colloque, mais c'est une démarche non moins nécessaire. Nous avons à faire dans l'histoire des figures la même révolution que les historiens du lexique après F. de Saussure et essayer d'atteindre une dimension structurale.

15 Cela dit, par rapport à cette attitude héritée des années 1970, qui nous enjoint à la fois de désacraliser l'Antiquité tout en envisageant le fait rhétorique dans ses contextes 
divers et ses divers modes d'organisation interne, on assiste aujourd'hui à un retour vers plus de connivence avec des cultures, la culture grecque en l'occurrence, dont nous avons reçu l'héritage et qui est constitutif de notre identité.

Nous empruntons à P. Payen $(2013: 166)$ cette phrase à la fois ouverte et circonspecte : "L'étude de l'Antiquité ne trouve pleinement sa finalité que si l'on mesure en quoi elle résonne parfois (pas toujours, certes) comme en écho à des problèmes ou des situations advenues plus tard dans le cours de l'histoire. L'Antiquité ne devient intelligible qu'à condition de prendre en compte - ne serait-ce qu'implicitement, dans le cheminement de la pensée, au moment où l'analyse se construit - les traditions issues de ce passé ou les analogies entre Anciens et Modernes, dès lors qu'elles sont instruites avec prudence et rigueur ".

17 Pour illustrer ce point, nous dirons que, personnellement, nous n'hésitons pas à relier la conception ancienne de la figure comme geste mental assimilé grâce à la répétition à la théorie moderne de la mémoire de travail à long terme. Dans les deux cas, il s'agit d'automatiser des procédures complexes pour parvenir à une compétence spontanée. L'intérêt de ce rapprochement est - par exemple - qu'il permet de nous réapproprier en connaissance de cause, et non plus de manière irrationnelle ou empirique, des méthodes d'enseignement très anciennes.

18 3) Mais nous devons aussi - pour en finir avec les prolégomènes - clarifier notre position sur les idées reçues que nous voulons dénoncer. Les idées reçues flottent dans l'air et certaines d'entre elles n'ont pas d'origine déterminée, même si l'on peut tenter de les comprendre. Le préjugé défavorable à la rhétorique tient sans doute à ce que l'on ne voit pas immédiatement l'intérêt d'apprendre ce que tout le monde sait faire, c'està-dire parler; en vertu d'un paralogisme, ce surapprentissage est associé à de la dissimulation ou au mensonge.

Certaines des idées reçues dont nous allons parler sont de cet ordre, mais certaines autres ont des sources parfaitement identifiables : la comparaison des noms de figure à ceux de "poissons zébrés de noir et d'argent ", c'est-à-dire le reproche adressé à la théorie des figures d'abuser d'une terminologie incompréhensible vient d'H. Morier (1981) dans l'introduction de son Dictionnaire de poétique et de rhétorique. Si nous parlons de "rage taxinomique ", tout le monde comprendra que nous pensons à R. Barthes (1985: 156).

20 Il existe aussi des idées reçues plus insidieuses : le projet initial de P. Fontanier, au début du XIX $x^{e}$ siècle, devait s'intituler Traité général des figures du discours, et ce n'est qu'à cause des programmes scolaires qui à l'époque affectaient l'étude des tropes à la classe de seconde et l'étude des figures non tropes à la classe de rhétorique que l'ouvrage a été divisé en deux parties publiées séparément, avant que G. Genette ne le réédite en $1977^{3}$ sous le titre Les figures $d u$ discours, plus respectueux du projet original. On voit bien quelle idée marque l'emploi de l'article défini et le singulier du mot discours: le discours recouvre toute énonciation quelle qu'elle soit, et les figures sont toutes les figures possibles.

21 Cette tendance à l'unification et à la systématisation, en vertu d'une sorte de finalisme implicite selon lequel la théorie serait en marche vers une sorte de vérité intangible, existe dans l'Antiquité tardive et pour les mêmes raisons pédagogiques, nous en parlerons, mais elle ne permet pas de rendre compte du processus d'invention de 
chaque pan de la doctrine et elle risque de nous priver de tous les apports de la démarche historique.

En d'autres termes, certains préjugés concernant les figures tiennent à ce qu'elles ont constitué non seulement un objet d'enquête historique mais une matière d'enseignement dogmatique, alors qu'il y a souvent plus à apprendre dans le processus de formation des doctrines, même lorsque le résultat est balbutiant, que dans l'examen des systèmes clos.

Après cette trop longue introduction, nous voudrions examiner quatre de ces idées qui nous semblent à la fois largement acceptées et trompeuses.

\section{Une terminologie absconse}

Que ce soit la comparaison avec les poissons exotiques opérée par H. Morier, ou l'exemple burlesque souvent donné des insultes du capitaine Haddock, ces lazzi illustrent la même critique d'une terminologie à la fois proliférante et absconse. Il ne s'agit pas tout à fait d'un préjugé, parce que nous sommes bien obligés de constater la complexité réelle du lexique relatif aux figures, mais nous sommes persuadé que cette complexité, une fois tournée en ridicule, sert de prétexte pour considérer ce savoir comme inutile et encombrant. Bref: il importe de sauver les figures de leur terminologie.

25 En disant tout d'abord que la responsabilité de ce jargon incombe, et c'est bien normal, à l'opacité d'une langue, le grec, que l'on n'apprend plus guère. Mais pour les hellénistes - et il en va de même en matière de lexique médical -, les noms de figure sont les plus transparents du monde: le klimax, appelé aussi gradation, n'est rien d'autre qu'une échelle, ce qui a l'avantage de faire voir immédiatement qu'on peut la monter ou la descendre, c'est-à-dire classer les mots dans un ordre d'intensité croissant ou descendant.

Plus intéressant, quand on passe la barrière de la langue, le fait que les noms de figure sont généralement des déverbatifs d'action, c'est-à-dire non seulement des mots transparents en grec mais des mots particulièrement concrets. La paraleipsis, c'est l'action qui consiste à presque laisser de côté. Dans le domaine moral, c'est le mépris des devoirs, qui signifie à la fois qu'on sait quel est son devoir et qu'on ne le fait pas. En rhétorique, le préfixe para- indique qu'on laisse, qu'on omet (c'est le sens du second élément du composé, leipei), mais en passant si près du référent que l'on fait deviner ce que l'on omet.

27 Ce qui est curieux, et qui mériterait une étude plus précise, c'est que le sème de proximité tend à disparaitre, si l'on en croit le dictionnaire de Liddell-Scott-Jones ${ }^{4}$ et que ce qui reste dans paraleipsis à date tardive c'est la simple idée d'omission, chez Athénée, par exemple, ou Plutarque, comme si le mot grec avait été contaminé par sa traduction latine prateritio.

Ce mot latin, d'où vient le français prétérition, indique tout simplement que l'on passe par-dessus ou au-delà de la mention du référent, c'est-à-dire qu'on l'omet.

29 Entre le fait de frôler la mention et la pure et simple omission, outre la perte de précision, on devine aussi qu'un changement de point de vue s'est opéré : dans un cas on épouse subjectivement l'intention de l'orateur qui parle, dans l'autre, on est plutôt dans la position du lecteur qui constate un manque dans un texte. 
30 Nous pourrions donner beaucoup d'exemples de noms de figures qui sont des noms d'action, ce qui montre le lien intime entre la doctrine des figures et l'énonciation orale, alors que nous privilégions depuis longtemps, dans le métadiscours sur les textes, comme G. Genette l'a observé, l'écrit et la perspective critique.

31 C'est l'occasion de citer un passage d'Isocrate très éclairant sur l'origine métaphorique de l'emploi du mot skhèma, que nous traduisons par figure :

" Ainsi, lorsqu'ils ont pris en main leurs élèves, les pédotribes leur enseignent les positions ( $\sigma x \eta ́ \mu \alpha \tau \alpha)$ que l'on a inventées pour la compétition ( $\left.\alpha_{\gamma} \gamma v^{\prime} \alpha\right)$; ceux qui s'occupent de philosophie font à leurs disciples un exposé complet des formes

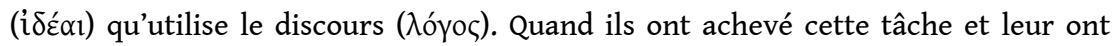
donné de l'expérience, ils recommencent à les exercer, les habituent au travail et les obligent à relier les uns aux autres chacun des éléments qu'ils ont appris, afin qu'ils les possèdent de façon plus sûre et que leurs opinions s'appliquent mieux aux événements » (Isocrate, Sur l'Échange, § 183-184).

32 Autrement dit, les formes que l'on donne au discours sont autant de postures, de positions, qui permettent d'obtenir l'avantage, comme à la lutte. Ce sont des techniques qui s'apprennent par la répétition. Citons aussi cet autre passage, où se trouve explicitée la pédagogie sous-jacente :

«Il faut d'abord que l'élève, outre les dons naturels qu'il doit posséder, apprenne les formes des discours et s'exerce à les pratiquer, et que le maître, d'autre part, soit capable de les expliquer avec assez de précision pour ne rien omettre de ce qui peut s'enseigner; pour le reste, il doit s'offrir lui-même en modèle tant et si bien que les élèves ayant reçu son empreinte et capables de l'imiter s'avèrent tout à coup des orateurs plus fleuris et plus gracieux que tous les autres » (Isocrate, Contre les Sophistes, § 17-18).

Les figures, ou formes du discours, sont conçues comme des techniques de combat que les élèves ne doivent pas seulement apprendre en théorie, mais surtout pratiquer, dans un constant échange entre la répétition et l'imitation du maitre. In fine, les empreintes mentales que sont devenues les figures permettent une soudaine transformation de l'élève en bon orateur. Tous les sportifs ont expérimenté cette conversion que procure soudainement la maitrise d'un geste.

Nous avons bien conscience d'avoir dévié par rapport au thème de la terminologie, mais il fallait expliquer cette prévalence des déverbatifs d'action dans les noms de figure, qui tient à tout un dispositif pédagogique. Nous ajouterons juste que les formes métaphorisées comme figures recouvrent indifféremment - c'est un point que nous n'avons pas le temps de développer ici - des procédés stylistiques et des contenus de pensée, et que les idées ou figures isocratiques sont les ancêtres du couple figure d'expression/figures de pensée. L'enjeu est de taille. Isocrate se considère non pas comme un rhéteur mais comme un philosophe et ces figures qu'il enseigne inlassablement ne sont pas les outils du parleur, mais ceux du citoyen : elles recouvrent à la fois des compétences oratoires et les compétences réflexives de l'acteur politique.

\section{La figure ornementale}

Un préjugé courant, accroché à un cliché comme celui des « fleurs de rhétorique » veut que les Anciens aient affecté aux figures une fonction purement ornementale. En réalité, il existe des figures ornementales et reconnues comme telles, attachées à la catégorie stylistique de beauté, en vertu du principe selon lequel «ce qui est beau à 
voir est aussi beau à dire » (Démétrios, 1993 : $\$ 174)$. C'est le cas de l'épiphonème décrit par Démétrios dans son traité Du Style (ibid. : § 106), qui donne en exemple un poème de Sappho évoquant la marche de jeunes bergers dans la montagne, indifférents aux fleurs, des jacinthes, qu'ils écrasent sous leurs pieds. Et Sappho d'enchainer sur une phrase nominale qui - selon Démétrios - n'apporte rien au sens du texte :

« et à terre, la fleur de pourpre... $~^{5}$

mais lui apporte, dit-il, « ornement et beauté ». Et il est vrai que sans rien apprendre de plus on assiste à la fois à un arrêt sur image et à un « zoom » sur le spectacle pathétique de la fleur écrasée. Mais la beauté est une catégorie stylistique parmi d'autres.

Par ailleurs, les rhéteurs anciens ont parfois subi des influences philosophiques qui les ont amenés à rejeter complètement la conception ornementale de la figure. Dans l'introduction du traité des figures d'Alexandros, on lit ceci :

« car il n'est pas facile non plus de trouver un discours qui soit sans figure, et s'il en va ainsi c'est par nécessité : le discours, en effet, est issu d'une impression de l'âme - c'est ce pour quoi il a été inventé, d'ailleurs, pour extérioriser les formes, les affections et, en général, les mouvements de celle-ci; or l'âme est toujours en mouvement et elle adopte de très nombreuses postures/figures ( $\sigma x \eta \mu \alpha \tau ı \sigma \mu o u ́ c)$ en vue de la mise en discours, définissant, conseillant, délibérant, subissant ou effectuant forcément un parmi tous les autres événements qui lui arrivent, si bien que, à l'imitation de ce qui se passe dans l'âme, le discours aura forcément une figure, quelle qu'elle soit » (Spengel, $1856: 11$ ).

On reconnait là ce que S. Aubert (2006) a appelé stoïcème, c'est-à-dire un bout de doctrine stoïcienne $e^{6}$ transplanté en rhétorique. La théorie sous-jacente est que l'âme est le réceptacle d'empreintes de la réalité qui la mettent en mouvement et le langage l'écho direct de ces mouvements, à savoir une série de positions ou de postures. De la même façon les Stoïciens ont conçu une rhétorique identique à la dialectique, c'est-àdire un mode de démonstration effectué à partir des représentations cataleptiques, soit des empreintes du réel distinguées des représentations fausses liées au souvenir, à l'imagination ou au délire, et approuvées par l'âme et pour cela considérées comme vraies.

On voit par là que la figure ornementale n'a aucun droit à subsumer toutes les conceptions anciennes de la figure. C'est ainsi que les rhéteurs grecs ont su dépasser la traditionnelle définition de la figure comme écart par rapport à l'usage, définition dont les linguistes du Groupe $\mu$ ont montré les impasses. On vient de constater, avec Alexandros, que certains rhéteurs considéraient toute énonciation comme figurée. Un autre théoricien des figures, nommé Tibérios (Spengel, 1856 : 57-82 ; Ballaira, 1968), a répertorié les figures employées par Démosthène. Un stylisticien du nom de Démétrios a répertorié les figures en fonction de différents " caractères $»^{7}$ du discours, figures de la grandeur, figures de la simplicité, ou en fonction de différentes illocutions : les figures de la séduction ou les figures de l'autorité. On voit bien ici que la norme n'est pas l'usage insaisissable mais la coïncidence entre un mode d'énoncé et une relation à l'auditeur du discours qui peut être générale et recouvrir un type d'illocution, ou personnelle, comme dans le cas de Démosthène.

41 On a même l'esquisse d'une définition de la figure comme purement structurale, dont la valeur n'apparait que dans un jeu d'opposition. D'où la possibilité pour des figures contraires, comme le mot composé ou la périphrase, de produire le même effet de grandeur, comme déjà, à l'époque hellénistique, le reconnaissait le rhéteur Démétrios 
(1993 : § 92). Cela tient non seulement au fait que la figure n'est pas un signifiant, mais aussi au fait que c'est son insertion dans un contexte qui lui donne, relativement, son efficace.

Pour faire un point rapide sur ces conceptions, nous citerons ici un passage de l'introduction à la récente édition du De ideis d'Hermogène (2012-2014) par Michel Patillon :

«Les figures sont au troisième rang dans l'ordre d'importance des composants du style, après les pensées et les mots, et elles ne sont étudiées dans le De ideis qu'en fonction de leur valeur stylistique. Il en résulte que toute forme d'expression susceptible d'avoir une telle valeur [...] entrera dans cette classe de figures. Or en vertu du système d'opposition des ideai (c'est-à-dire les catégories d'illocutions décrites dans le traité), il suffit qu'une forme de l'expression s'oppose à une autre forme pour être remarquable. [...] Au plan théorique et doctrinal, le fait important est que l'écart pris en compte ici n'est pas un écart entre une forme d'expression donnée et une forme théoriquement normale » (CRhet. IV : XLIV-XLV).

Nous en avons assez dit pour faire comprendre que les «fleurs de rhétorique » ne sauraient rendre compte des doctrines antiques des figures.

\section{Une métaphore sans sémiologie}

Passons à un troisième préjugé selon lequel les Anciens n'auraient pas réussi à appréhender la dimension proprement sémantique des figures. L'origine de cette idée est claire : on se souvient du fait que P. Ricœur reprochait à Aristote, sur le terrain de la métaphore, d'en rester au mécanisme du transfert, ou de la substitution, et d'ignorer la dimension sémantique de ce type d'énoncé remarquable. Et il est vrai que le philosophe se préoccupe surtout de découvrir les rapports entre les objets ${ }^{8}$. Pour lui, la métaphore - qui correspond en fait mutatis mutandis à l'ensemble de la catégorie ultérieure de trope - consiste à désigner un objet du nom d'un autre objet qui entretient avec le premier objet un lien particulier en termes de catégorie ou d'analogie. Mais si la métaphore nous apprend quelque chose sur lui, elle ne désigne en tout et pour tout qu'un objet. Ni le processus de mise en rapport des deux objets, ni le versant sémantique de cette opération n'intéressent Aristote.

L'un des traités les plus curieux du Corpus rhetoricum s'intitule De inventione (CRhet., 2012 , t. III, $1^{\text {re }}$ partie). On y lit ce passage :

«L'échange ( donné (ن் sujet donné et au sujet introduit de l'extérieur, ce que les grammairiens appellent aussi

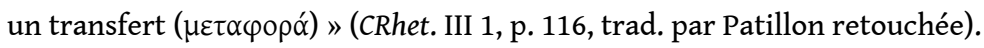

Le commentaire de Michel Patillon souligne l'importance théorique d'un passage comme celui-ci : on pourrait dire que c'est l'acte de naissance de la sémiologie, c'est-àdire d'une analyse de la signification comme phénomène spécifique, à cette différence près, par rapport à la plupart des théories modernes, et conformément à ce que nous avons déjà observé dans les théories anciennes, que le fonctionnement est décrit du point de vue de l'usage qu'en fait l'orateur. Essayons d'être plus précis.

Le premier progrès est de prendre comme unité de fonctionnement de la métaphore non pas le mot mais le "sujet donné ", c'est-à-dire le contenu d'une séquence discursive de longueur variable, celle qui est prise en compte dans le fonctionnement 
de la métaphore. Ce découpage donne un caractère dynamique à ce qui est une opération complexe. M. Patillon poursuit :

« À la différence d'Aristote, dont la théorie de la métaphore étudie le rapport entre le nom métaphorique et le nom absent dont il a pris la place [personnellement, je dirais plutôt entre les deux objets désignés], notre auteur pose d'emblée le nom métaphorique dans son ambivalence (...). On a moins là une définition de la métaphore qu'une description de son fonctionnement du point de vue de l'usage qu'en font les rhéteurs. (...) Ce point de vue est aussi ce qui fait la justesse de la théorie de notre auteur, puisque la métaphore résulte d'un certain fonctionnement linguistique du discours, non seulement avec émergence d'un sens, ce qui est vrai de tout discours, mais d'un sens qui violente le code sémantique. » (op. cit., p. 187)

Pour le dire autrement

«... le progrès décisif accompli ici est que tout risque de concevoir la métaphore comme une substitution se trouve écarté. (...) Il n'y a rien là qui suggère une présence et une absence, comme si un nom avait pris la place d'un autre; il y a présence double: dans son emploi métaphorique le nom s'intègre, ici, maintenant, aux deux sujets. En d'autres termes, il renvoie simultanément à deux modèles référentiels étrangers l'un à l'autre. D'où un enrichissement sémique dans ce contexte du nom métaphorique et une tension dans le discours. » (op. cit., p. C)

On pourrait parler aussi de dynamique herméneutique.

De qui est ce texte important, de quand date-t-il? Probablement de la période impériale, plus précisément de la fin $\mathrm{du}_{\mathrm{II}}^{\mathrm{e}}$ ou du début du $\mathrm{III}^{\mathrm{e}}$ siècle ap. J.-C. L'une des meilleures hypothèses d'attribution désigne comme auteur un certain Aspasios de Ravenne, connu pour son talent d'improvisation, ce qui n'est pas indifférent, eu égard à la perspective adoptée ici dans l'évocation du processus métaphorique.

Dans la notice de Wikipedia sur la métaphore, il n'y a rien dans la partie « définition » entre Aristote et Fontanier, et certaines définitions récentes, celles de M. Meyer et de P. Bacry, par exemple, sont encore très marquées par l'idée de substitution.

\section{La rage taxinomique}

52 Là encore, l'origine du reproche est connue. Ce que met R. Barthes sous cette formule est relativement élaboré. Elle vise à dénoncer une tentative impossible qui est de figer des énonciations à priori innombrables, et à plaquer des critères de classements maniaques et inappropriés sur des listes, ce qui revient à maquiller maladroitement l'incapacité à ordonner véritablement les figures.

La première chose à dire est que les listes de figures ont existé bien sûr, mais qu'une bonne partie des figures anciennes ont d'abord été dotées d'une sorte d'unité fonctionnelle. Prenons comme exemple la catégorie des figures "gorgianiques ».

Leur liste varie, mais elles comprennent généralement l'antithèse, la parisose, c'est-àdire l'égalité de deux côla en nombre de syllabes, et la paromoiose, ou échos sonores de côlon à côlon. Ces figures ont un caractère très voyant, d'où leur association au nom de Gorgias, selon un processus que Marie-Pierre Noël (1999) a décrit en suivant l'histoire de la critique littéraire relative à ce sophiste. Mais sur ce chemin, il y a une étape importante, représentée par la Rhétorique d'Aristote. On y apprend que ces figures ont été réunies autour de la notion de période, unité de respiration, unité d'énonciation, unité de pensée, et même unité de raisonnement. 
Pour Aristote en effet, le substrat de la pensée est la perception. La formulation de la pensée est donc plus ou moins favorable à la compréhension du message. De là une théorie qui codifie le découpage de la chaine parlée en unités relativement « digestes », pas trop longues, les périodes, dotées d'une unité de sens et découpées en côla, ou membres, dotés d'une relative autonomie, et dont les relations puissent être aisément intelligibles. C'est à cela que servent les figures de parallélisme, d'échos sonores, et d'antithèse sémantique, d'autant que pour Aristote la période était faite de deux membres et que, disait-il, les « contraires sont très identifiables et plus identifiables encore quand ils sont à côté l'un de l'autre ». On peut donc faire l'hypothèse que cette binarité de la période faisait d'elle un instrument privilégié de la réfutation, car la réfutation - toujours selon Aristote - procède par la juxtaposition des opposés 9 .

56 Autrement dit - et nous nous arrêterons là sur un sujet qui mériterait de plus amples développements - les figures gorgianiques sont un avatar tardif d'une catégorie de figures dont la fonction commune est de découper dans la chaine parlée des unités aisément intelligibles, porteuses de raisonnement. On est bien loin de la figure anecdotique, dont ni la fonction, ni les synergies éventuelles ne seraient identifiées.

Mais le meilleur antidote aux jugements de R. Barthes sur la rage taxinomique est probablement l'œuvre d'un rhéteur du nom de Phoebammon: son traité n'est pas encore disponible en français, nous y travaillons, mais on peut le lire en grec dans le troisème volume des Rhetores graeci de L. Spengel (1856:41-56).

On y découvre, au carrefour de la philosophie, de la grammaire et de la rhétorique, un classement structural des figures. Son principe est la quadripertita ratio: addition, soustraction, mutation, métathèse (ou permutation). Cette séquence finie d'opérations est bien connue : elle sert à structurer la néologie selon les Stoïciens ${ }^{10}$. En clair, il s'agissait pour eux de décrire de façon systématique la façon dont s'était défait, au fil du temps, l'accord entre les mots et les choses, puisque, on le sait, cette école philosophique était restée fidèle à ce que l'on appelle cratylisme, c'est-à-dire à l'idée d'un lien direct, naturel, entre les mots et les objets qu'ils désignent.

Que la quadripartition soit utilisée par les Stoïciens est une chose, qu'elle soit d'origine stoïcienne en est une autre. C'est une thèse souvent défendue mais non prouvée. F. Desbordes (2007:60) souligne à juste titre le rôle probable des catégories de la physique d'Aristote: le mouvement et le changement s'y produisent soit selon la quantité (augmentation ou diminution), soit selon la qualité (altération), soit selon le lieu (translation).

Quoi qu'il en soit, les champs d'application de la quadripertita ratio sont nombreux: outre l'étymologie, l'orthographe, le barbarisme, le métaplasme (ou altération de la forme d'un mot, alignée par Consentius sur le barbarisme ${ }^{11}$ ), la composition (ou adaptation du mot à son contexte phonique ${ }^{12}$ ), la paronomase, le solécisme et autres fautes, la métrique.

61 Mais Phœbammon est le seul à organiser clairement et explicitement l'ensemble des figures de cette manière, même si des tentatives en ce sens paraissent avoir été faites avant lui ${ }^{13}$.

62 Comment se présente le traité ? Chez Phœbammon, les figures sont opposées, en tant que jeu sur la combinatoire, aux tropes qui opèrent pour leur part sur l'axe paradigmatique et procèdent de substitutions ponctuelles. Divisées en figures de 
pensée et figures de mots, elles forment un ensemble rendu cohérent par la nature des quatre opérations effectuées parallèlement dans l'un et l'autre domaines.

Ce qui est remarquable est que le système ainsi produit évoque mutatis mutandis le classement proposé aux derniers temps de la rhétorique restreinte, dans la Rhétorique générale du Groupe $\mu(1982)^{14}$. Certes, le système proposé par les savants de Liège est plus compréhensif (depuis les éléments non signifiants jusqu'au texte) et se fonde sur des connaissances linguistiques beaucoup plus fines. Mais les analogies n'en sont pas moins frappantes, principalement sur le parallélisme entre la forme de l'expression et la forme du contenu ${ }^{15}$ et sur la nature des opérations universelles. Les linguistes belges parlent d'opérations "substantielles", où toute "transformation " se réduit à une addition, à une suppression ou à une suppression-adjonction d'unités; et des opérations "relationnelles ", à savoir la permutation, quelconque ou par inversion (Groupe $\mu$, 1982: $45 \mathrm{sq.)}$.) La parenté entre les opérations est indiscutable.

Phœbammon présente un autre intérêt. On se souvient que pour R. Barthes toutes les énonciations sont singulières et donc impossibles à systématiser. Mais citons le rhéteur ancien :

«Que l'ensemble des figures de pensée n'est pas modifié par la façon de dire : car si l'on reformule cette façon de dire, la même pensée demeure, par exemple à propos d'un absent, je demande: va-t-il venir? Si je suis dans l'inquiétude, je pose la question autrement : untel vient, n'est-ce pas? Je peux dire encore : Acceptera-t-il, aura-t-il le culot de venir? Se donnera-t-il la peine de venir? Au travers de toutes ces formulations, la pensée, à savoir le but de l'interrogation, reste la même. Ces figures de pensée se divisent en deux catégories : les unes concernent la personne, les autres le discours.

Il y en a dix-huit au total. Trois concernent la seule personne: l'apostrophe, la question, l'investigation. L'apostrophe, c'est quand nous adressons le discours au juge ou à l'adversaire. La question et l'investigation diffèrent en ce que la question reçoit une réponse brève, par exemple :

Par les dieux, ce n'est pas ton nourricier, Onésime?

à quoi répondent ces quelques mots : "mais si, tout à fait", alors que l'investigation reçoit une réponse plus étendue, ainsi :

Qui, issu de qui parmi les hommes?

Pour y répondre, Glaukos a dû remonter dans sa généalogie.

Il faut savoir que, si les figures de pensée sont au nombre de dix-huit, les figures de mots sont au nombre de vingt-six. On doit savoir que toutes les figures se font selon quatre modes ou causes, par soustraction, par addition, par métathèse, par mutation ». (Rhetores graeci, tome III, Leipzig, 1856, p. 44-45 Spengel; traduction personnelle)

F. Desbordes a montré qu'en fait les énonciations elles aussi ont des traits récurrents dans ce qui peut les séparer de la forme simple, ou de base. Elle propose par exemple comme schéma de base " $\mathrm{A}$ dit $\mathrm{x}$ (énoncé) à $\mathrm{B}$ ». L'apostrophe s'écarte de cette forme de base en ce que le locuteur, soudain, feint de dire à l'adversaire ce qu'il dit au juge. Dans le texte que nous venons de citer, on voit que Phœbammon, en distinguant les figures de pensée selon qu'elles concernent la personne ou le discours, rend possible une théorie de ce genre. En clair, avec l'apostrophe, on aura une figure de pensée indépendante de sa formulation - à l'instar de l'interrogation - qui joue non pas sur le discours mais sur la mutation du destinataire. Et il peut s'agir indifféremment non pas d'un énoncé, mais d'une énonciation future, qui sera ainsi inscrite dans un système clos. La tâche, évidemment, n'est pas achevée. 
66 Phœbammon ${ }^{16}$ est le nom d'un (ou plusieurs ?) sophiste(s) des $\mathrm{V}^{\mathrm{e}} / \mathrm{VI}^{\mathrm{e}}$ siècles de notre ère. Si l'on se range à la thèse unitaire, on peut penser qu'il était d'origine égyptienne. Peut-être fut-il le professeur du fameux moine Jean Moschos (l'auteur du Pré spirituel, traduit chez Migne dernièrement par V. Déroche et $\mathrm{C}$. Bouchet) à l'époque où ce dernier, à la fin du vi ${ }^{e}$ siècle, vivait à Antinoupolis. Il composa un commentaire à Thucydide, perdu, un commentaire aux États de la cause d'Hermogène, lui aussi perdu mais dont certaines parties ont survécu dans les Prolégomènes à Hermogène plus tardifs (Walz, 1832: vol. VIII, 522 sq.; vol. VII, 40 sq.). On a conservé en revanche son commentaire sur le traité hermogénien des Catégories stylistiques et plusieurs citations byzantines conduisent à penser que le commentaire aux Catégories stylistiques transmis sous le nom de Syrianus sont en fait de lui (ibid. : 67, 5 ; Gärtner, 1972).

Nous ne saurions conclure sans recommander chaudement la lecture des traités récemment édités dans la collection Budé. Tous ne sont pas d'une lecture distrayante, il faut en convenir, mais l'historien ou le curieux des théories du langage y trouvera du grain à moudre.

\section{BIBLIOGRAPHIE}

AUBERT, S. (2006) : Per dumeta. Recherches sur la rhétorique des Stoïciens à Rome, de ses origines grecques jusqu'à la fin de la République, thèse, Université Paris IV-Sorbonne, nov.

BACRY, P. (2010) : Les figures de style, Paris, Belin.

BALlAIRA, G. (éd.) (1968) : Tiberii de figuris demosthenicis libellus, Rome, Ateneo.

BARTHES, R. (1985) [1970] : «L'ancienne rhétorique. Aide-mémoire », in : L'Aventure sémiologique, Paris, Éd. Le Seuil, p. 85-165.

BROWN, P. (1983) [1976] : Genèse de l'Antiquité tardive, Paris, Gallimard.

CHIRON, P. (1999) : « La période chez Aristote », in : P. Büttgen, S. Diebler \& M. Rashed (éds), Théories de la phrase et de la proposition, de Platon à Averroès, Paris, Presses de l'ENS, p. 103-130. - (2002) : « La métaphore encore », La Tribune internationale des langues vivantes, 31, mai, p. 21-30. CHIRON, P. \& LÉvy, C. (éds) (2010) : Les Noms du Style dans l'Antiquité classique, Louvain/Paris, Peeters.

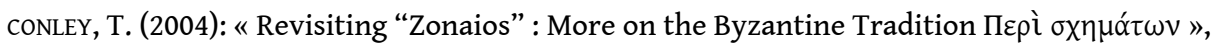
Rhetorica, 22, p. 257-268.

DÉMÉTRIos (1993) : Du Style, texte établi et trad. par P. Chiron, révisé par G. Aujac, Paris, Éd. Les Belles Lettres.

- (2007) : Lo stile, éd. prés. par N. Marini, Rome, Éd. di stora et letteratura.

DENYS D’HALICARNASSE (1981): Opuscules rhétoriques, t. III, La composition stylistique, t. établi et traduit par G. Aujac et M. Lebel, Paris, Éd. Les Belles Lettres.

Pratiques, 165-166 | 2015 
DESBORDES, F. (2006) [1986] : «L'énonciation dans la rhétorique antique : les "figures de pensée" », in : F. Desbordes, Scripta varia. Rhétorique antique et littérature latine, textes réunis par G. Clerico \& J. Soubiran, Louvain/Paris, Peeters, p. 109-120.

- (2007) [1983] : « Le schéma "addition, soustraction, mutation, métathèse" dans les textes anciens ", in : F. Desbordes, Idées grecques et romaines sur le langage, textes réunis par G. Clerico, B. Colombat \& J. Soubiran, avec Lyon, ENS Éd., p. 55-63.

DOUAY, F. \& SERMAIN, J.-P. (2007) : Pierre « Émile » Fontanier, la rhétorique et les figures de la Révolution à la Restauration, Québec, Presses de l'université Laval (chapitre 2 en ligne : www.lpl-aix.fr/ fulltext/2898, consulté le 9 avril 2014).

GÄRTNER, H. (1972) : Der Kleine Pauly, t. IV, Munich, Druckenmüller.

GROUPE $\mu$ (1982) [1970] : Rhétorique générale, Paris, Éd. Le Seuil.

HERMOGÈNE (2012-2014) : Corpus rhetoricum, 5 tomes, éd. et trad. par M. Patillon, Paris, Éd. Les Belles Lettres.

MEYER, M. (2008) : Principia Rhetorica, Une théorie générale de l'argumentation, Paris, Éd. Fayard.

MORIER, H. (1981) [1961] : Dictionnaire de poétique et de rhétorique, $3^{\mathrm{e}}$ éd. augmentée et entièrement refondue, Paris, Presses universitaires de France.

NOËL, M.-P. (1999) : « Gorgias et l'invention des GORGIEIA SCHÈMATA », Revue des études grecques, 112, p. 193-211.

PAYEN, P. (2013) : «Meurtre et homicide, guerre et souillure : infliger la mort en Grèce ancienne. À propos de Bernard Eck, La Mort rouge. Homicide, guerre et souillure en Grèce ancienne, Paris, Les Belles Lettres, 2012 - 448 p. : bibliogr., index. - (Études Anciennes, ISSN : 1151-826X : série grecque ; 145). ISBN : 978.2.251.32682.5 », Revue des études anciennes, 115, p. 165-174.

SPENGEL, L. (éd.) (1856) : Rhetores graeci, t. III, Leipzig, Teubner, p. 7-40.

VoIGT, E.-M. (1971) : Sappho et Alcaeus: Fragmenta, Amsterdam, Athenaeum-Polak and Van Gennep. WALZ E. C. (1832) : Rhetores graeci, volumes VII et VIII, Stuttgart, J. G. Cottae.

\section{NOTES}

1. La traduction française, sous le titre Genèse de l'Antiquité tardive, a été publiée chez Gallimard en 1983.

2. En ligne : https://fr.wikipedia.org/wiki/Figure_de_style, consulté le 9 avril 2014.

3. Sur cette publication et ses versions préparatoires, voir F. Douay et J.-P. Sermain (2007).

4. En ligne : http://stephanus.tlg.uci.edu/lsj/\#eid=1\&context=lsj, consulté le 9 avril 2014.

5. Sappho [?], fr. 105b dans Voigt (1971).

6. En l'occurrence Diogène Laërce VII 49.

7. Sur cette terminologie, voir P. Chiron et C. Lévy (2010).

8. Pour une mise au point sur cette question, $c f$. P. Chiron (2002).

9. Voir Aristote, Rhétorique, III, 1410 a 1419 sq. Sur l'ensemble de la question, voir P. Chiron (1999).

10. Cf. Varron, De lingua latina, V, 6 ; VI, 2 ; VII, 1 ; Quintilien, 1, 6, 32. Varron cite comme sources principales de sa méthode étymologique les stoïciens Chrysippe et Antipater.

11. Grammatici Latini, p. 387 Keil V.

12. Cf. Denys d'Halicarnasse (1983).

13. Cf. Plutarque, De Homero, II, p. 15 sq. Kindstrand ; Quintilien, 9, 3, 27 et F. Desbordes (2007: 59$)$. 
14. Voir aussi F. Desbordes (2006, notamment p. 119).

15. Unités de signifiant $v s$ unités de signifié, $c f$. Groupe $\mu(1982: 30)$.

16. Cf. Pauly-Wissowa, Realencyclopädie, XX, 1, München 1941, col. 326-343 [W. Stegemann]; H. Gärtner (1972, col. 794-795). Les scolies Des figures sont dans C. Walz (1832, p. 497-519) et L. Spengel (1856 : 41-56).

\section{RÉSUMÉS}

Nous essayons dans cet article de présenter les principaux traits marquants des théories anciennes des figures et d'attirer l'attention sur quelques erreurs fréquemment commises dans leur interprétation. Nous sélectionnons quatre de ces préjugés, afin de pouvoir développer à loisir leur origine, leur signification et les moyens de s'en guérir : l'obscurité de la terminologie ; l'idée selon laquelle les figures se réduiraient à une fonction ornementale ; l'absence de véritable sémiologie dans la théorie ancienne de la métaphore; et enfin la "rage taxinomique ", selon la formule de R. Barthes, c'est-à-dire la manie qu'auraient eu les rhéteurs anciens de décrire et de classer des énonciations par nature illimitées.

We try in this paper to present the main features of the theories of figures/stylistic devices in Antiquity, and to point out some frequent mistakes in their interpretation. We choose four of these prejudices in order to develop at length their origin, their meaning and the way to cure them: the obscurity of terminology; the ornamental character of figures; the absence of semiology in the ancient theories of metaphor; and finally what Barthes used to call "rage taxinomique " (taxonomical fury), that is to say the obsession of describing and classifying a verbal reality which is not within our grasp.

\section{INDEX}

Mots-clés : rhétorique, figures, procédés stylistiques, histoire des théories du langage, Antiquité

Keywords : rhetoric, figures, stylistic devices, history of the theories of language, Antiquity

\section{AUTEUR}

\section{PIERRE CHIRON}

Lis (EA 3495), Université Paris-Est Créteil Val-de-Marne, Institut universitaire de France 